\title{
Measuring disease activity to predict therapeutic outcome in Graves' ophthalmopathy
}

\author{
C. B. Terwee*, M. F. Prummelt, M. N. Gerding†, G. J. Kahaly§, F. W. Dekker* $¥$ and W. M. Wiersinga† \\ Departments of ${ }^{\star}$ Clinical Epidemiology and Biostatistics and $†$ Endocrinology and Metabolism, Academic Medical Centre, \\ University of Amsterdam, $\ddagger$ Department of Clinical Epidemiology, Leiden University Medical Centre, the Netherlands and \\ $\S$ Department of Endocrinology/Metabolism, Gutenberg-University Hospital, Mainz, Germany
}

\section{Summary}

Objective The concept of disease activity in Graves' ophthalmopathy (GO) might explain why as many as one-third of patients do not respond to immunosuppressive treatment, because only patients in the active stage of disease are expected to respond. The hypothesis was adopted that a parameter used to measure disease activity should be able to predict a response to immunosuppressive treatment. The aim of this study was to develop a multivariate prediction model in which all previous tested activity parameters are integrated.

Design and Patients We included 66 consecutive patients with untreated moderately severe GO who had been euthyroid for at least 2 months. All patients were treated with radiotherapy.

Measurements Treatment efficacy after 6 months follow-up was used as the primary outcome measure. Disease severity and 15 different disease activity parameters were assessed before treatment. Univariate and multivariate logistic regression models were used to predict response (model 1) or no change (model 2).

Results In multivariate analyses, we found that duration of GO, soft tissue involvement, elevation, soluble interleukin-2 receptor (sIL-2R), soluble CD30 (sCD30), eye muscle reflectivity and octreotide uptake ratio were significant predictors of a response to radiotherapy. Gender, duration of GO, soft tissue involvement, eye muscle reflectivity, IL-6 and urinary glycosaminoglycan (GAG) excretion were significant predictors of no change upon radiotherapy. Prognostic score charts were developed for use in clinical practice to calculate the probability of response (model 1) and the probability of no change (model 2) for each new patient. Finally we used a combination of both models to define a recommended treatment modality for each individual patient, based on both the predicted probabilities of response and no change. We were able to identify the correct treatment (based on a comparison with the observed response) in $89 \%$ of the patients.

Conclusions Although we strongly recommend that our results should be confirmed in other studies, our findings are the first

Correspondence: Dr C. B. Terwee, Institute for Research in Extramural Medicine, VU University Medical Center, Van der Boechorststraat 7, 1081 BT Amsterdam, the Netherlands. Tel.: 3120 4448187; Fax: + 3120 4448181; E-mail: cb.terwee.emgo@med.vu.nl evidence for the idea that disease (in)activity should determine which kind of treatment should be used.

(Received 31 July 2003; returned for revision 22 September 2003; finally revised 1 June 2004; accepted 1 September 2004)

\section{Introduction}

The concept of disease activity, originating from observations on the natural history of Graves' ophthalmopathy (GO) by Rundle and his co-workers, is attractive because it might explain why as many as one-third of patients do not respond to immunosuppressive treatment. ${ }^{1-5}$ According to Rundle's curve ${ }^{6}$ it can be expected that only patients in the active stage of disease will respond to corticosteroids or radiotherapy, whereas patients with inactive disease will not respond. ${ }^{7}$ The generally found response rate to immunosuppression of about $65 \%$ can thus be explained by the failure to distinguish active from inactive disease.

Several methods have been described to determine disease activity, ${ }^{7,8}$ However, it is not easy to assess whether a proposed parameter measures disease activity, as comparison with a gold standard (a biopsy of the extraocular eye muscles) is not feasible. For this reason, the hypothesis was adopted that a parameter that is measuring disease activity should be able to predict a response to immunosuppressive treatment. Consequently, the predictive value of a proposed activity parameter is considered to be a measure of the ability of the parameter to measure disease activity.

In previous studies, we and others assessed the predictive value of several potential activity parameters to predict response to radiotherapy, ${ }^{8}$ such as duration of the eye disease, ${ }^{8-10}$ the Clinical Activity Score (CAS), ${ }^{11}$ urinary glycosaminoglycan (GAG) excretion, ${ }^{12,13}$ serum cytokine levels, ${ }^{14,15}$ serum levels of TSH-receptor autoantibodies, ${ }^{16}$ eye muscle reflectivity assessed by orbital ultrasonography, ${ }^{17,18} \mathrm{~T} 2$ (relaxation time from MRI) values of the eye muscles calculated from magnetic resonance imaging (MRI) scans ${ }^{19,20}$ and the orbital uptake of ${ }^{111}$ indium-labelled octreotide. ${ }^{21-24}$ Some of these parameters could predict a response to treatment to some extent, while others could predict a nonresponse, also to some extent. These results suggest that a combination of different parameters may be 
needed to accurately predict response to treatment. Therefore, the aim of this study was to develop a multivariate prediction model in which all previously tested activity parameters are integrated. To do this, the patients were selected not by using any activity parameter but only on the basis of the severity of their eye disease.

\section{Methods}

\section{Patients}

We included 66 consecutive patients who qualified for orbital radiotherapy (20 Gy, given in 10 fractions of 2 Gy over a 2-week period) because of evident restriction of eye muscle motility (usually with diplopia) and/or proptosis of $\geq 25 \mathrm{~mm}$. We did not include patients with optic nerve involvement (they were considered to be too severe) or with contraindications for radiotherapy (e.g. diabetes mellitus). Only patients who had not received prior therapy for their eye disease (except for local lubricants) were included and all had to be euthyroid for at least 2 months, as defined by normal values for free T4 $(10 \cdot 0-22 \cdot 0 \mathrm{pmol} / \mathrm{l})$ and total T3 $(1 \cdot 30-2 \cdot 45 \mathrm{nmol} / \mathrm{l})$ in the absence of elevated TSH levels $(>4.0 \mathrm{mU} / \mathrm{l})$. The study was approved by the Medical Ethics Committees of the Academic Medical Centre in Amsterdam and all patients gave their written informed consent.

\section{Assessment of therapeutic outcome}

Potential markers for disease activity (see below) were recorded at baseline and related to the therapeutic outcome at 6 months after start of treatment using predefined major and minor response criteria. Our major and minor criteria were adapted from those used by Bartalena et al. (1998). Major criteria were: (1) a change of $\geq 8^{\circ}$ in mono-ocular duction in the most affected direction of gaze (mostly elevation) measured using a modified hand perimeter; ${ }^{25}$ (2) a change of $\geq 1$ grade in the degree of diplopia: intermittent (present only occasionally), inconstant (in certain directions of gaze only) or constant (present in primary position of gaze); ${ }^{26}$ (3) a change in pinhole visual acuity of $\geq 1$ line(s) on the Snellen chart. ${ }^{27}$ Minor criteria were: a change of $\geq 2 \mathrm{~mm}$ in lid aperture; a change of $\geq 2 \mathrm{~mm}$ in proptosis; a change of $\geq 1$ grade in soft tissue involvement on colour slides. ${ }^{28}$ A response to treatment was defined as 'very good' in the case of an improvement in at least two major criteria, as 'good' in the case of an improvement in one major criterion, as 'fair' in the case of an improvement in two minor criteria, as 'no change' in the case of no changes or a change in only one minor criteria, and as 'worse' in the case of a deterioration in at least one major or two minor criteria. The categories 'very good', 'good' and 'fair' were considered a response, the categories 'no change' and 'worse' were considered a nonresponse. All patients were examined by the same ophthalmologist. Because the orbits are not always equally affected, we used measurements of the most affected eye.

In addition, mono-ocular eye muscle motility in all four directions of gaze was used to calculate a range of motion as the surface (in $\mathrm{mm}^{2}$ ) of the square made up by the ductions in the four directions of gaze $\left(1^{\circ}=1 \mathrm{~mm}\right)$.

\section{Assessment of disease activity}

The following potential parameters of disease activity were measured before the start of radiotherapy: (1) the duration of the ophthalmopathy was assessed in months since the onset of the eye disease, as perceived by the patient; (2) the CAS; ${ }^{11,29}$ (3) GAG excretion was measured in two 24-h urine collections using high-performance liquid chromatography (HPLC) and analysed in the laboratory of the Department of Endocrinology/Metabolism, Gutenberg-University Hospital, Mainz, Germany (Prof. G.J. Kahaly); total GAG content and the subfractions of chondroitin sulfate (CA), dermatan sulfate (DS) and hyaluronic acid (HA) were determined as described previously; ${ }^{13,30}$ (4) a panel of immunoregulatory molecules was measured in serum by an enzyme-linked immunosorbent assay (ELISA) as described elsewhere, ${ }^{15}$ consisting of interleukin-6 (IL-6), IL-6-receptor, IL-1 receptor antagonist (IL-1RA), soluble IL-2 receptor (sIL-2R), tumour necrosis factor-alpha receptor I and II (TNF $\alpha$ $\mathrm{RI}$ and II), and soluble CD30 (sCD30); (5) autoantibodies against the thyrotrophin receptor (TSH-R) were measured as thyrotrophin binding inhibitory immunoglobulins (TBII) using the TRAH assay (Brahms Diagnostica, Berlin, Germany), and as TSH-R stimulating immunoglobulins (TSI) using a CHO cell line stably transfected with the human TSH-R; ${ }^{17}$ (6) eye muscle reflectivity was assessed by an experienced independent investigator with standardized A-mode ultrasonography of both orbits, using an Ophthascan-S unit, as described earlier. ${ }^{20}$ The muscle showing the lowest reflectivity (in \%) was used in the analysis. ${ }^{7}$ The T2 relaxation times on MRI were measured as described earlier ${ }^{20}$ and the muscle with the lowest T2 time (in ms) was used in the analysis. ${ }^{8}$ The uptake at $4 \mathrm{~h}$ after injection of ${ }^{111}$ indium-diethylenetriaminepentaacetic acid (DTPA)-octreotide was measured quantitatively as the mean of eight orbital single photon emission computed tomography (SPECT) scan images and expressed as a ratio $v s$. the uptake in a temporal area. ${ }^{31}$ The orbit with the highest uptake ratio was used in the analysis.

\section{Statistical analyses}

Univariate and multivariate logistic regression models were used to predict two binary outcomes from pretreatment clinical variables: one model (model 1) was developed to predict response to radiotherapy ( $v s$. nonresponse, including worse); a second model (model 2) was developed to predict no change upon radiotherapy ( $v s$. response or worse). This second model will be used to select those patients in whom immediate surgery can be performed. Two approaches were used for each model: an optimal approach, using as many predictive (in)activity parameters as possible, and a practical approach, in which expensive, or laborious parameters were removed or substituted by cheaper or more easily available parameters.

Variables were entered in the model in the order in which they become available. First, a basic model was built, based on general variables (age, sex, duration of GO, and parameters of disease severity). Age was included as a continuous variable, after checking the linearity assumption. Duration was included as a dichotomous outcome (cut-off 16 months) in the model to predict response, and as a continuous variable in the model to predict no change, as this resulted in the best predictive model. Additionally, the potential 
parameters of disease activity were added one by one (no automated stepwise procedure was used) and checked for their additional predictive value. Only variables that contributed significantly to the predictive value of the models (defined as a $P$-value of $<0 \cdot 10$ ) were retained. For missing values within variables (four missing for sIL2R; four missing for sCD30; 21 missing for octreotide uptake), the sample median was imputed. All possible interaction terms were tested among the significant $(P<0 \cdot 10)$ variables in the models. Interaction terms that contributed significantly to the predictive value of the models (defined as a $P$-value of $<0 \cdot 10$ ), were retained.

The probability of response (model 1 ) or no change (model 2) was estimated for each patient, based on the regression coefficients $(b)$ of each of the variables included in the model $\left(X_{1-n}\right)$, using the following formula of the regression model: $Y=a+b_{1} X_{1}+b_{2} X_{1}+\ldots$ $+b_{n} X_{n}$. The probability of response (model 1 ) or no change (model 2 ) was defined as $P=\mathrm{e}^{Y} /\left(1+\mathrm{e}^{Y}\right)$. For each cut-off point of this probability, sensitivity and specificity can be calculated and plotted in a receiver operator characteristic (ROC) curve. The area under the ROC curve (AUC) was calculated as a measure of the discriminative ability of the model. ${ }^{32}$ An area of 0.50 indicates no discriminative ability, an area of 1.0 indicates perfect discriminative ability. An area of at least $0 \cdot 80$ is usually considered satisfactory.

\section{Results}

We included 66 patients in the study: 35 (53\%) responded to radiotherapy; 31 (47\%) did not respond, of whom 26 (39\%) were classified as 'no change'. In Table 1 the clinical characteristics of the patients are presented for the whole group, as well as for each category of treatment response. All patients remained stable in their visual acuity during the course of the study (data not shown).

Univariate logistic regression analyses showed that patients with a duration of GO of more than 16 months have a 10 times lower probability of response to radiotherapy [odds ratio (OR) $0 \cdot 1,95 \%$ confidence interval (CI) $0 \cdot 05-0 \cdot 4]$, that is a $10(95 \%$ CI $2 \cdot 5-20)$ times larger probability of nonresponse (Table 2, model 1). Patients with moderate soft tissue involvement have a $3 \cdot 7(1 \cdot 1-11 \cdot 8)$ times larger probability of response, and patients with severe soft tissue involvement have a $5 \cdot 4(1 \cdot 0-28 \cdot 5)$ times larger probability of response. Patients with a decreased elevation have a $1 \cdot 7(1 \cdot 1-2 \cdot 4)$ times larger probability of response for every $8^{\circ}$ less elevation. In the analyses aimed at predicting no change (Table 2, model 2), it was found that patients with a duration of GO of more than 16 months have a $14 \cdot 5(4 \cdot 1-51 \cdot 7)$ times larger probability of'no change' to radiotherapy. All other parameters had insignificant or borderline significant odds ratios for the prediction of response or no change.

In multivariate analyses, we found that duration of GO, soft tissue involvement and elevation were significant predictors of a response to radiotherapy. The discriminative ability of this practical model, which was restricted to easy-to-obtain parameters, was 0.82 (95\% CI 0.73-0.92), which means that from a group of pairs of patients, each pair consisting of a responder and a nonresponder, the model would be able to correctly identify the responders in $82 \%(73-92 \%)$ of the cases. If more expensive or laborious parameters were added to the model, sIL-2R, sCD30, eye muscle reflectivity and octreotide uptake ratio were of significant additional value in the prediction of response (Table 3). No significant interactions were found. The discriminative ability of this optimal model was $0 \cdot 89(0 \cdot 82-0 \cdot 97)$. Duration of GO and soft tissue involvement were also significant predictors of no change in the multivariate analyses. In addition, we found that gender and eye muscle reflectivity were of significant additional value in the prediction of no change. The discriminative ability of this relatively simple (practical) model was $0 \cdot 86(0 \cdot 77-$ $0 \cdot 95$ ). If more expensive or laborious parameters were added to the model, IL-6 and GAG excretion were of significant additional value in the prediction of no change (Table 3 ). No significant interactions were found. The discriminative ability of the optimal model was $0 \cdot 93(0 \cdot 87-0 \cdot 99)$.

For each patient, the probability of response (model 1) and the probability of no change (model 2) were estimated from both the practical and the optimal models. Using practical model 1, we found that all patients with an estimated probability of response of less than $22 \%$ were nonresponders. These comprised 14 patients. Using optimal model 1, all patients with an estimated probability of response of less than $20 \%$ were nonresponders. These comprised 16 patients. Using the practical model 2, we found that all patients with an estimated probability of no change of more than $80 \%$ were not changed. These comprised 10 patients. Using the extended, optimal model, all except one patient with an estimated probability of no change of more than $80 \%$ were not changed. These comprised 15 patients.

To make the models useful in clinical practice, a prognostic score chart was developed for the practical models 1 and 2, based on rounded regression coefficients. These score charts are presented in Figs 1 and 2 , and can be used to calculate the individual probability of response (Fig. 1) or no change (Fig. 2) upon radiotherapy for a new patient.

For example, a patient presenting with a duration of GO of 20 months, with mild soft tissue involvement and an elevation of $24^{\circ}$ receives a score of $-1.9-1.8+8=4.3$ points. From Fig. 1, the estimated probability of response is about $30 \%$. On the other hand, a patient presenting with a duration of GO of 6 months, with severe soft tissue involvement and with an elevation of only $8^{\circ}$ scores $1 \cdot 6-$ $0 \cdot 6+8=9$ points, with an estimated probability of response of almost $100 \%$.

In Figs 3 and 4 we present the predicted probabilities of response and the predicted probabilities of no change for each patient, using a combination of both optimal models (Fig. 3) or both practical models (Fig. 4). In Figs 5 and 6 we present a comparison of the optimal and practical models for the predicted probability of response (Fig. 5) and for the predicted probability of no change (Fig. 6).

Finally, in Tables 4 and 5 we present the predicted probabilities of response and the predicted probabilities of no change for each patient, using a combination of both optimal models (Table 4) or both practical models (Table 5 ). In these tables we also present the observed response to radiotherapy. In addition, a treatment modality is recommended for each individual patient based on these predicted probabilities (discussed below).

\section{Discussion}

We found that duration of GO, soft tissue involvement, elevation, sIL-2R, sCD30, eye muscle reflectivity and octreotide uptake ratio were significant predictors of a response to radiotherapy in a 


\begin{tabular}{|c|c|c|c|c|c|c|c|}
\hline & \multicolumn{6}{|l|}{ Response } & \multirow[b]{2}{*}{$P$-value $\$$} \\
\hline & All & Very good & Good & Fair & No change & Worse & \\
\hline Number (\%) & $66(100)$ & $13(20)$ & $18(27)$ & $4(6)$ & $26(39)$ & $5(8)$ & \\
\hline Age $^{*}$ & $53(10)$ & $54(9)$ & $49(12)$ & $51(7)$ & $56(9)$ & $50(12)$ & $0 \cdot 18$ \\
\hline Gender (F/M) & $45 / 21$ & $10 / 3$ & $13 / 5$ & $3 / 1$ & $16 / 10$ & $3 / 2$ & $0 \cdot 27$ \\
\hline Smoking (yes/no) & $32 / 34$ & $3 / 10$ & $11 / 7$ & $2 / 2$ & $12 / 14$ & $4 / 1$ & $0 \cdot 20$ \\
\hline \multicolumn{8}{|l|}{ Disease severity } \\
\hline Lid aperture $(\mathrm{mm})^{*}$ & $11 \cdot 8(2 \cdot 3)$ & $11 \cdot 2(2 \cdot 7)$ & $12 \cdot 3(2 \cdot 4)$ & $10 \cdot 5(1 \cdot 8)$ & $11 \cdot 6(1.9)$ & $14 \cdot 0(1 \cdot 3)$ & $0 \cdot 09$ \\
\hline $\begin{array}{l}\text { Soft tissue involvement } \\
(0 / \mathrm{a} / \mathrm{b} / \mathrm{c})\end{array}$ & $0 / 20 / 36 / 10$ & $0 / 0 / 9 / 4$ & $0 / 5 / 12 / 1$ & $0 / 1 / 1 / 2$ & $0 / 12 / 13 / 1$ & $0 / 2 / 1 / 2$ & $0 \cdot 02$ \\
\hline Proptosis $(\mathrm{mm})^{*}$ & $19 \cdot 5(3 \cdot 2)$ & $19 \cdot 2(3 \cdot 0)$ & $19 \cdot 0(2 \cdot 7)$ & $18 \cdot 3(2 \cdot 5)$ & $19 \cdot 7(3 \cdot 7)$ & $22 \cdot 4(2 \cdot 7)$ & $0 \cdot 26$ \\
\hline Elevation $(\mathrm{deg})^{*}$ & $14 \cdot 4(13 \cdot 6)$ & $11 \cdot 4(14 \cdot 8)$ & $7 \cdot 3(11 \cdot 9)$ & $16 \cdot 8(6 \cdot 2)$ & $18 \cdot 4(13 \cdot 3)$ & $25 \cdot 0(9 \cdot 2)$ & 0.02 \\
\hline Range of motion $\left(\mathrm{mm}^{2}\right)^{*}$ & $2282(1117)$ & $1785(1070)$ & $2077(992)$ & $1828(1028)$ & $2634(1184)$ & $2808(834)$ & $0 \cdot 11$ \\
\hline Diplopia $(0 / \mathrm{a} / \mathrm{b} / \mathrm{c})^{* *}$ & $3 / 9 / 19 / 35$ & $0 / 2 / 6 / 5$ & $1 / 1 / 4 / 12$ & $0 / 2 / 1 / 1$ & $1 / 3 / 7 / 15$ & $1 / 1 / 1 / 2$ & $0 \cdot 63$ \\
\hline \multicolumn{8}{|l|}{ Disease activity } \\
\hline Duration GO (months) $\dagger$ & $17(4-240)$ & $12(4-35)$ & $13(6-80)$ & $18(10-60)$ & $30(5-240)$ & $12(6-34)$ & $0 \cdot 002$ \\
\hline CAS $\dagger$ & $3(0-7)$ & $4(2-7)$ & $3(0-5)$ & $4(2-7)$ & $2(0-7)$ & $5(2-6)$ & 0.09 \\
\hline $\begin{array}{l}\text { (GAG) excretion } \dagger \\
(\mathrm{DS} \mathrm{mg} / 24 \mathrm{~h})\end{array}$ & $5 \cdot 4(0 \cdot 2-15 \cdot 6)$ & $8 \cdot 2(0 \cdot 2-11)$ & $5 \cdot 6(0 \cdot 9-15 \cdot 6)$ & $3 \cdot 4(1 \cdot 6-5 \cdot 4)$ & $5 \cdot 5(0 \cdot 3-10 \cdot 8)$ & $2 \cdot 4(0 \cdot 4-6 \cdot 8)$ & $0 \cdot 13$ \\
\hline IL-6 (ng/l)† & $2 \cdot 0(0 \cdot 6-23 \cdot 4)$ & $2 \cdot 3(0 \cdot 7-12 \cdot 1)$ & $2 \cdot 2(0 \cdot 6-23 \cdot 4)$ & $2 \cdot 5(1 \cdot 0-5 \cdot 4)$ & $1 \cdot 9(0 \cdot 9-4 \cdot 8)$ & $3 \cdot 8(1 \cdot 1-10 \cdot 0)$ & $0 \cdot 58$ \\
\hline IL-6R $(\mathrm{ng} / \mathrm{l}) \dagger$ & $35390(22280-52920)$ & $34560(22920-48840)$ & $34180(22280-51100)$ & $39480(35390-42640)$ & $35715(25080-52920)$ & $25360(23380-49620)$ & $0 \cdot 50$ \\
\hline TNF- $\alpha$ RI (ng/l) $\dagger$ & $1064(702-1778)$ & $1064(767-1436)$ & $1025(822-1373)$ & $1056(876-1184)$ & $1091(702-1697)$ & $1064(941-1778)$ & $0 \cdot 90$ \\
\hline TNF- $\alpha$ RII $(\mathrm{ng} / \mathrm{l}) \dagger$ & $3171(1823-6311)$ & $3232(1823-5898)$ & $3184(2099-4061)$ & $3167(2733-3556)$ & $2999(1856-6311)$ & $3182(2616-5122)$ & $0 \cdot 96$ \\
\hline IL-1RA (ng/l)† & $347(180-1836)$ & $334(180-444)$ & $350(188-1225)$ & $336(294-350)$ & $353(197-972)$ & $347(321-1836)$ & $0 \cdot 51$ \\
\hline sIL-2R (ng/l)† & $1230(540-2524)$ & $1138(952-2524)$ & $1302(616-2417)$ & $1164(942-1230)$ & $1227(540-2048)$ & $1491(1230-2344)$ & $0 \cdot 37$ \\
\hline $\mathrm{sCD} 30(\mathrm{U} / \mathrm{ml}) \dagger$ & $44(18-153)$ & $49(18-109)$ & $41(29-153)$ & $39(26-49)$ & $43(23-92)$ & $44(22-61)$ & $0 \cdot 72$ \\
\hline $\mathrm{TSI}(\mathrm{U} / \mathrm{ml}) \dagger$ & $31(16-130)$ & $44(21-130)$ & $26(18-107)$ & $46(21-57)$ & $33(18-130)$ & $42(16-130)$ & $0 \cdot 12$ \\
\hline TBII $(\mathrm{U} / 1) \dagger$ & $12(2 \cdot 5-400)$ & $45(2 \cdot 5-274)$ & $7 \cdot 5(2 \cdot 5-215)$ & $20(12-28)$ & $4 \cdot 8(2 \cdot 5-112)$ & $14(2 \cdot 5-400)$ & $0 \cdot 13$ \\
\hline US number & 56 & 10 & 16 & 2 & 24 & 4 & \\
\hline $\begin{array}{l}\text { US lowest eye muscle } \dagger \\
\text { reflectivity (\%) }\end{array}$ & $37(9-58)$ & $37(10-58)$ & $27(9-49)$ & $33(20-46)$ & $39(17-54)$ & $37(21-43)$ & $0 \cdot 32$ \\
\hline MRI number & 64 & 13 & 17 & 4 & 25 & 5 & \\
\hline MRI longest T2 time $(\mathrm{ms}) \dagger$ & $165(73-250)$ & $166(110-211)$ & $168(114-250)$ & $194(133-199)$ & $140(73-216)$ & $193(155-214)$ & $0 \cdot 18$ \\
\hline Octreoscan number & 45 & 8 & 12 & 4 & 17 & 4 & \\
\hline Octreotide uptake ratio $4 \mathrm{~h} \dagger$ & $2 \cdot 1(1 \cdot 0-3 \cdot 8)$ & $1 \cdot 8(1 \cdot 3-3 \cdot 4)$ & $2 \cdot 2(1 \cdot 4-3 \cdot 8)$ & $3 \cdot 0(1 \cdot 9-3 \cdot 2)$ & $1 \cdot 8(1 \cdot 0-2 \cdot 9)$ & $1 \cdot 8(1 \cdot 7-2 \cdot 3)$ & $0 \cdot 14$ \\
\hline
\end{tabular}

${ }^{\star}$ Mean (SD).

$\dagger$ Median (range).

$\$$ Most affected eye.

$\$ \chi^{2}$ for linear association (categorical data) or one-way analysis of variance (ANOvA) (continuous data).

$\mathbf{g} 0=$ absent; $\mathrm{a}=\operatorname{minimal} ; \mathrm{b}=$ moderate; $\mathrm{c}=$ marked.

${ }^{*} 0$ = no diplopia; $\mathrm{a}=$ intermittent; $\mathrm{b}=$ inconstant $\mathrm{c}=$ constant

DS, dermatan sulfate; laboratory of Prof. G Kahaly, Mainz, Germany. 
Table 2. Univariate odds ratios

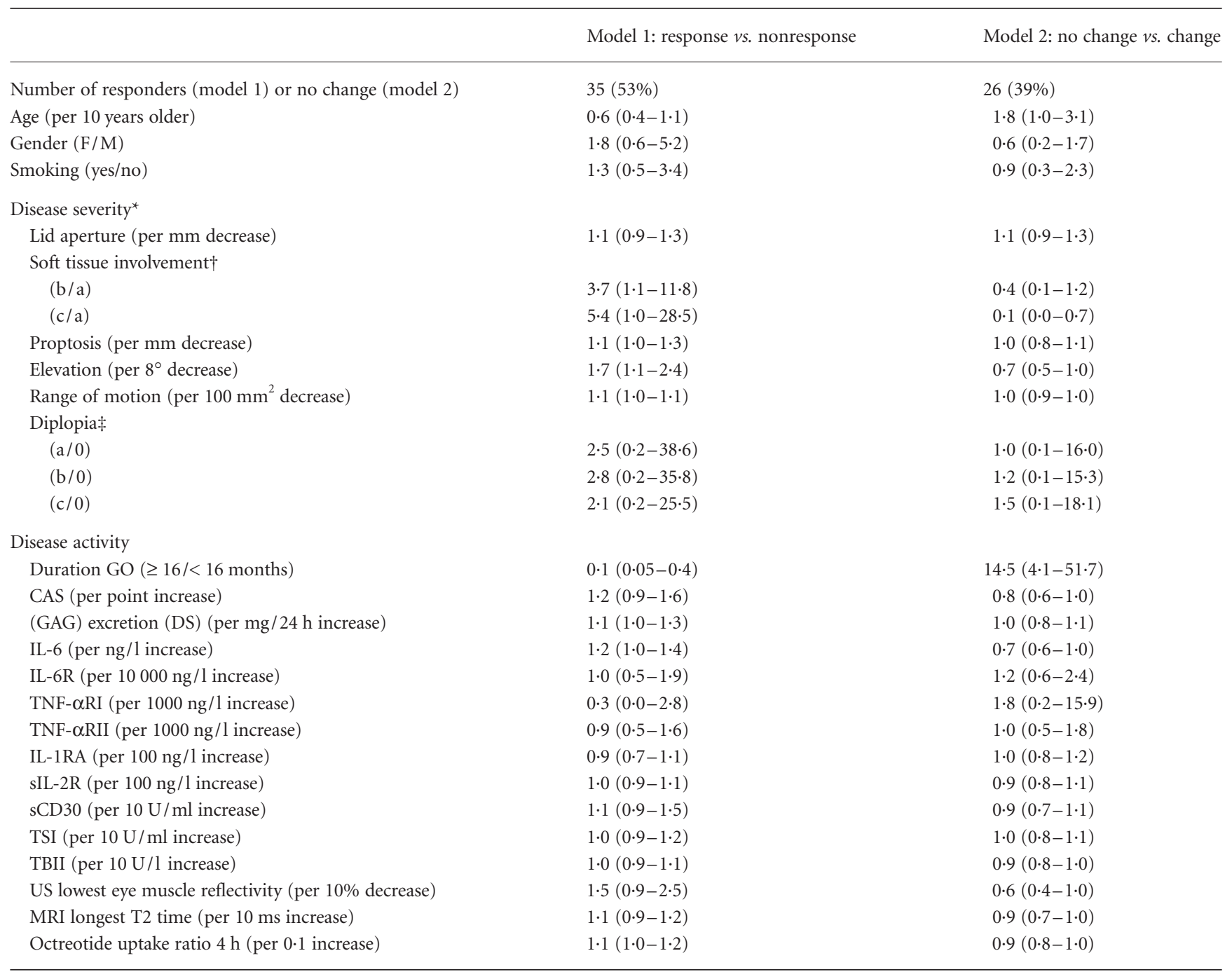

${ }^{\star}$ Most affected eye.

$\dagger 0=$ absent; $\mathrm{a}=$ minimal; $\mathrm{b}=$ moderate; $\mathrm{c}=$ marked.

$\neq 0=$ no diplopia; $\mathrm{a}=$ intermittent; $\mathrm{b}=$ inconstant; $\mathrm{c}=$ constant .

DS, dermatan sulfate; laboratory of Prof. Dr G Kahaly, Mainz, Germany.

\begin{tabular}{|c|c|c|}
\hline Prognostic factor & & Score \\
\hline \multirow{2}{*}{ Duration GO } & $<16$ months & 0 \\
\hline & $\geq 16$ months & -1.9 \\
\hline \multirow[t]{3}{*}{ Soft tissue involvement } & a & 0 \\
\hline & $\mathrm{b}$ & 1.5 \\
\hline & c & 1.6 \\
\hline \multirow[t]{3}{*}{ Elevation } & per $8^{0}<10$ & 0.6 \\
\hline & 0 & 0 \\
\hline & per $8^{0}>0$ & -0.6 \\
\hline Always add & & 8 \\
\hline Total score & & $\ldots$ \\
\hline
\end{tabular}

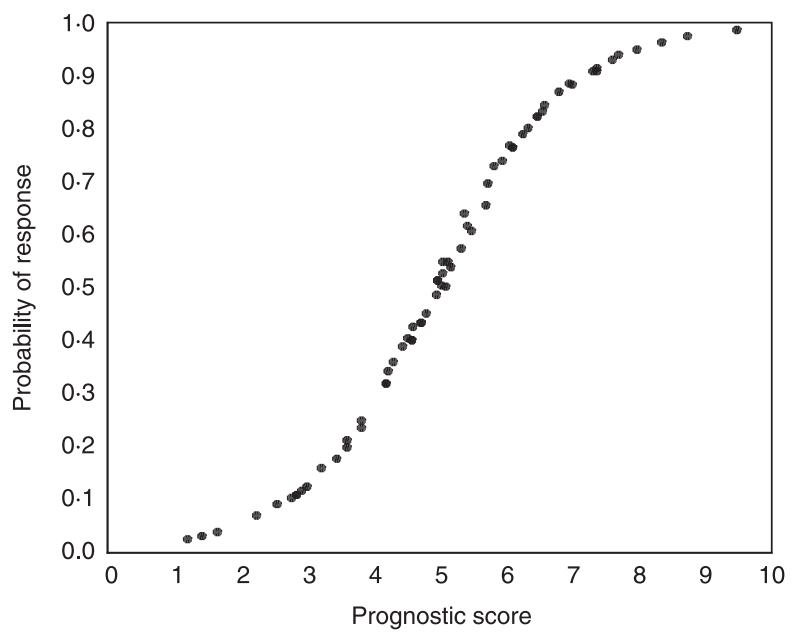

Fig. 1 Prognostic score chart for the prediction of response (practical approach). 
Table 3. Mutivariate odds ratios for the prediction of response vs. nonresponse (optimal approach)

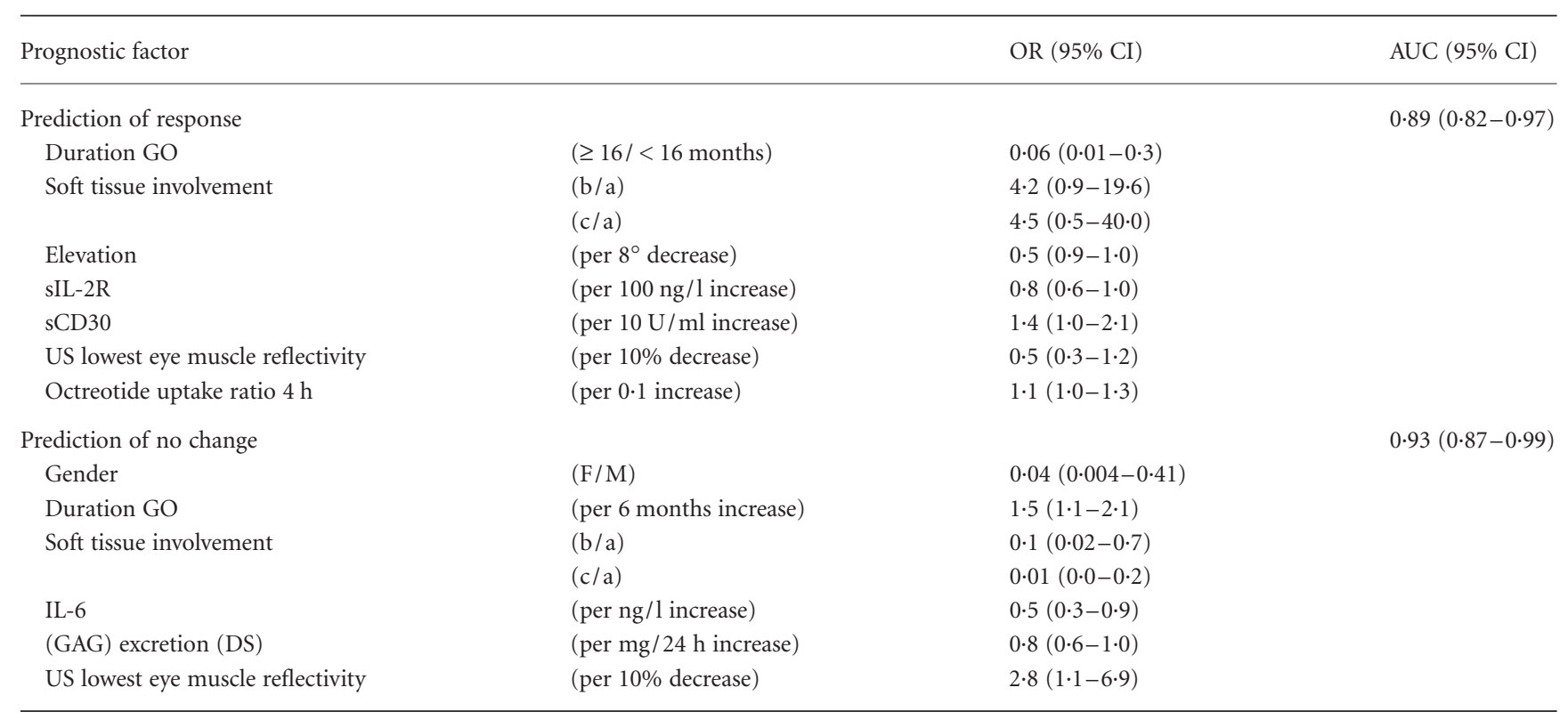

AUC, area under the ROC curve.

\begin{tabular}{|c|c|c|}
\hline Prognostic factor & & Score \\
\hline Duration GO & per 6 months & 0.3 \\
\hline \multirow[t]{3}{*}{ Soft tissue involvement } & a & 0 \\
\hline & $\mathrm{b}$ & $-1 \cdot 7$ \\
\hline & c & $-3 \cdot 3$ \\
\hline \multirow[t]{2}{*}{ Gender } & Female & 0 \\
\hline & Male & $-1 \cdot 6$ \\
\hline Ultrasound & per $10 \%$ & 0.7 \\
\hline Always add & & 6 \\
\hline Total score & & $\ldots$ \\
\hline
\end{tabular}

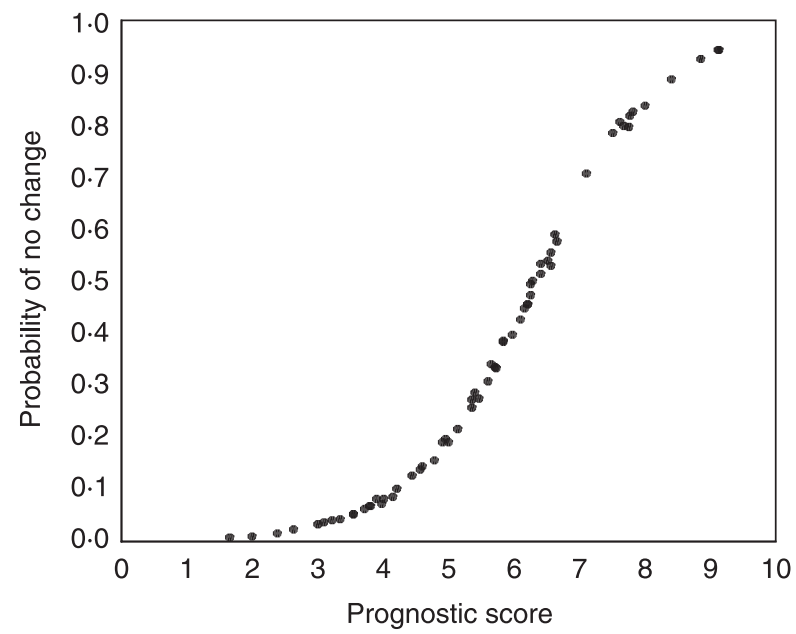

Fig. 2 Prognostic score chart for the prediction of no change (practical approach).

multivariate model. Duration of GO, soft tissue involvement and IL6 were significant predictors of no change in multivariate analyses. The discriminative ability of the practical models (using only easy available parameters) and also of the optimal models (using all potential predictive variables) was reasonable, with AUCs above 0.70.

It may seem surprising that characteristics of disease severity were included in the models. We included these variables because the aim was to predict response to treatment as best as possible based on all information available. The predictive value of severity parameters such as soft tissue involvement and elevation is understandable. Soft tissue involvement was judged based on signs of eyelid swelling, chemosis, redness of the conjunctiva and eyelids, all signs that are related to disease activity and are included in the CAS. ${ }^{11,29}$ This might explain why the CAS as such did not enter the models, as it was partly covered in the class 2 signs. In addition, more severe eye disease is more likely to improve by itself than is less severe ophthalmopathy. This explains why elevation was included in our models: a patient with more severe motility disturbances has more to gain from treatment. Finally, patients with severe ophthalmopathy are more likely to be at the top of Rundle's curve, and thus to be active. ${ }^{6}$

The relatively high discriminative ability of our models indicates that these models can be used to discriminate between groups of patients with a high or low probability of response. For example, if all patients with a probability of response of less than $50 \%$, based on the optimal model 1 , would have been treated with radiotherapy, only $8 / 33(24 \%)$ patients would have responded (Table 4 ). On the other hand, if all patients with a probability of response of more than $50 \%$, based on this model, would have been treated with radiotherapy, 27/33 (82\%) patients would have responded. Using model 2, we found that if all patients with a probability of no change of less than 


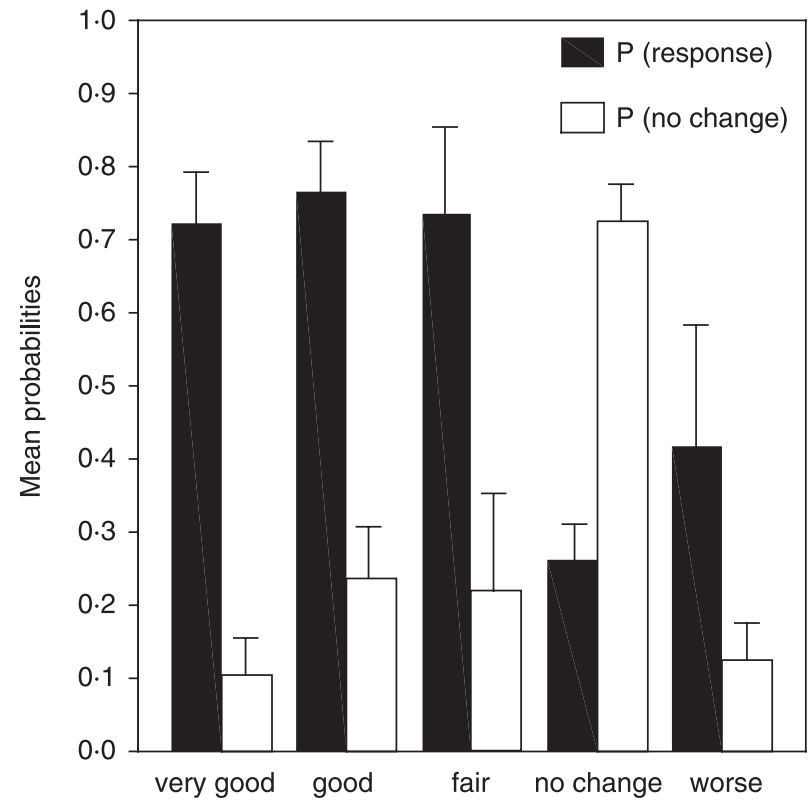

Fig. 3 Mean probabilities (SE) of response and no change, based on the optimal models.

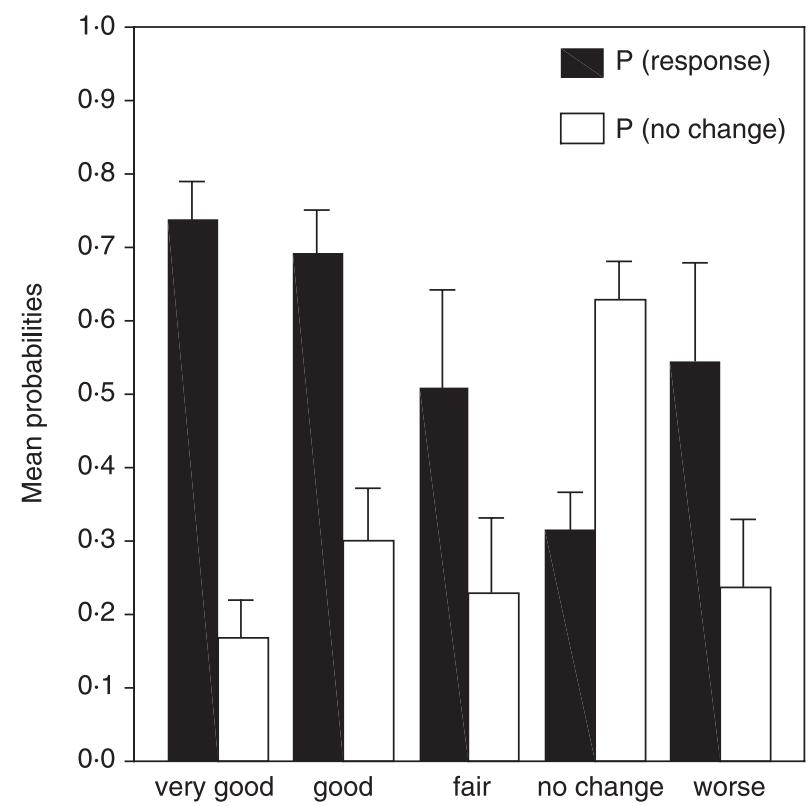

Fig. 4 Mean probabilities (SE) of response and no change, based on the practical models.

$30 \%$ would have been treated with radiotherapy, only $2 / 35(6 \%)$ patients would not have been changed. However, if all patients with a probability of no change of more than $30 \%$ would have been treated with radiotherapy, 24/31 (77\%) patients would not have been changed. By contrast, the CAS itself has a limited discriminative ability (AUC $0 \cdot 60$, data not shown). If we had used model 1 , based only on the CAS, to treat all patients with a probability of response of less than 50\%, 9/25 (36\%) would have responded, and if we had treated all patients with a probability of more than $50 \%, 26 / 41(63 \%)$ would have responded, which gives no benefit in comparison with the usual response rates. ${ }^{1-5}$

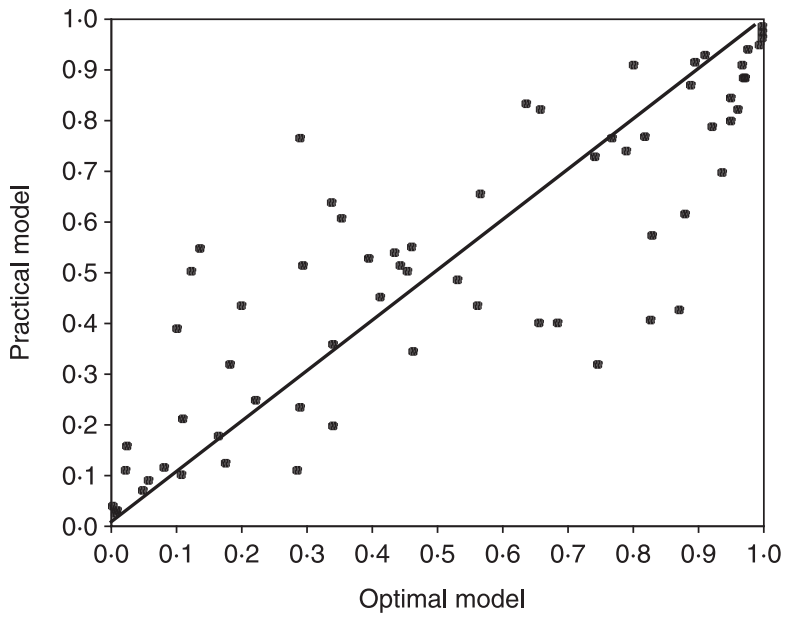

Fig. 5 Optimal model $v s$. practical model for the probability of response.

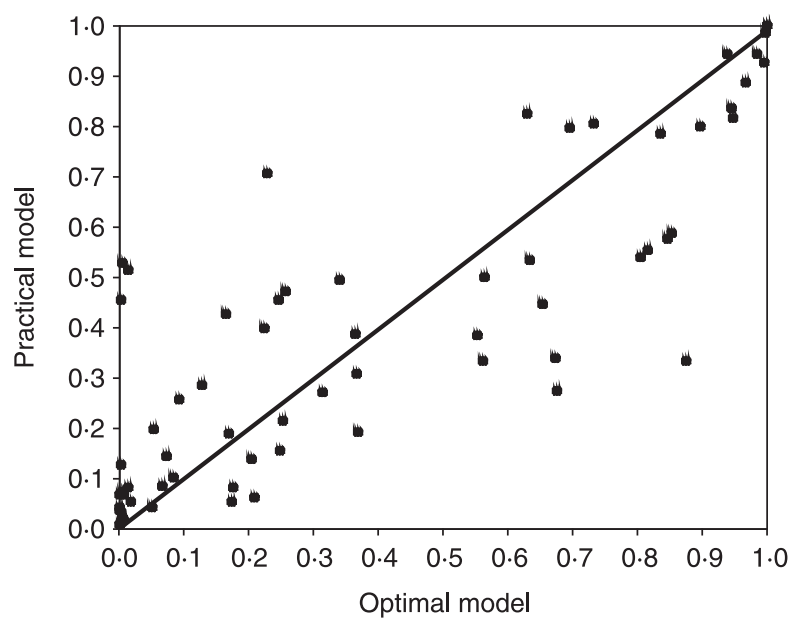

Fig. 6 Optimal model $v s$. practical model for the probability of no change.

The choice to use either model 1 (aimed at predicting response to radiotherapy) or model 2 (aimed at predicting no change) is not straightforward. If we only treat patients who are likely to respond, based on model 1, we would increase the response rate of radiotherapy to, for example, $82 \%$, depending on our cut-off point, as indicated in the example above. However, among the untreated patients (with a low probability of response), there would still be a minority ( $24 \%$ in our example) that would have responded. More importantly, there would be some patients in the untreated group that would have deteriorated upon radiotherapy (three patients in our study). These latter patients, in particular, should not yet be submitted to rehabilitative surgery, as they would probably still have active disease. For this reason, we also tried to predict inactivity or no change to radiotherapy, to be able to select those patients in whom immediate surgery can be performed, thereby shortening the period of disabling symptomatology. However, if we do not want to select any patient who would have been changed upon radiotherapy, we could at best select $12 / 66(18 \%)$ inactive patients with our models. 
Table 4. Individual predicted probabilities of response (optimal model 1) or no change (optimal model 2), observed response, and recommended treatment based on these probabilities

\begin{tabular}{|c|c|c|c|c|c|c|c|c|c|}
\hline Patient & $P$ (response) & $P$ (no change) & Response $^{\star}$ & Treatment $\dagger$ & Patient & $P$ (response) & $P$ (no change) & Response $^{*}$ & Treatment $\dagger$ \\
\hline 1 & $0 \cdot 00$ & $0 \cdot 98$ & 4 & S & 34 & $0 \cdot 53$ & $1 \cdot 00$ & 4 & S \\
\hline 2 & $0 \cdot 01$ & $1 \cdot 00$ & 4 & $S$ & 35 & $0 \cdot 56$ & $0 \cdot 25$ & 4 & $\mathrm{R}$ \\
\hline 3 & $0 \cdot 01$ & $0 \cdot 22$ & 5 & $\mathrm{R}$ & 36 & $0 \cdot 57$ & $0 \cdot 17$ & 2 & $\mathrm{R}$ \\
\hline 4 & $0 \cdot 02$ & $0 \cdot 94$ & 4 & $S$ & 37 & $0 \cdot 64$ & $0 \cdot 17$ & 2 & A \\
\hline 5 & $0 \cdot 03$ & 0.63 & 4 & $S$ & 38 & $0 \cdot 66$ & $0 \cdot 90$ & 2 & A \\
\hline 6 & $0 \cdot 05$ & $1 \cdot 00$ & 4 & $S$ & 39 & $0 \cdot 66$ & $0 \cdot 07$ & 2 & A \\
\hline 7 & 0.06 & $1 \cdot 00$ & 4 & $S$ & 40 & $0 \cdot 68$ & $0 \cdot 25$ & 3 & A \\
\hline 8 & $0 \cdot 08$ & $0 \cdot 95$ & 4 & $S$ & 41 & $0 \cdot 74$ & $0 \cdot 00$ & 1 & A \\
\hline 9 & $0 \cdot 10$ & $0 \cdot 34$ & 4 & $\mathrm{R} \ddagger$ & 42 & $0 \cdot 75$ & $0 \cdot 73$ & 2 & A \\
\hline 10 & $0 \cdot 11$ & 0.97 & 4 & $\mathrm{~S}$ & 43 & $0 \cdot 77$ & $0 \cdot 01$ & 1 & A \\
\hline 11 & $0 \cdot 11$ & $0 \cdot 36$ & 4 & $\mathrm{R} \ddagger$ & 44 & $0 \cdot 79$ & $0 \cdot 00$ & 5 & A \\
\hline 12 & $0 \cdot 12$ & 0.65 & 4 & $S$ & 45 & $0 \cdot 80$ & $0 \cdot 13$ & 1 & A \\
\hline 13 & $0 \cdot 14$ & $0 \cdot 01$ & 5 & $\mathrm{R}$ & 46 & $0 \cdot 82$ & $0 \cdot 18$ & 5 & A \\
\hline 14 & $0 \cdot 16$ & 0.95 & 4 & $S$ & 47 & $0 \cdot 83$ & $0 \cdot 01$ & 3 & A \\
\hline 15 & $0 \cdot 18$ & 0.82 & 4 & S & 48 & $0 \cdot 83$ & $0 \cdot 23$ & 2 & A \\
\hline 16 & $0 \cdot 18$ & $0 \cdot 84$ & 4 & $S$ & 49 & $0 \cdot 87$ & $0 \cdot 37$ & 2 & A \\
\hline 17 & $0 \cdot 20$ & 0.56 & 1 & $\mathrm{R}$ & 50 & $0 \cdot 88$ & 0.09 & 4 & $\mathrm{~A} \ddagger$ \\
\hline 18 & $0 \cdot 22$ & 0.67 & 4 & $S$ & 51 & $0 \cdot 89$ & $0 \cdot 00$ & 1 & A \\
\hline 19 & $0 \cdot 28$ & 0.63 & 4 & $S$ & 52 & $0 \cdot 89$ & 0.68 & 4 & $A \ddagger$ \\
\hline 20 & 0.29 & 0.87 & 4 & $S$ & 53 & $0 \cdot 91$ & 0.05 & 1 & A \\
\hline 21 & $0 \cdot 29$ & $0 \cdot 26$ & 2 & $\mathrm{R}$ & 54 & 0.92 & $0 \cdot 00$ & 1 & A \\
\hline 22 & $0 \cdot 29$ & 0.00 & 2 & $\mathrm{R}$ & 55 & $0 \cdot 94$ & $0 \cdot 00$ & 1 & A \\
\hline 23 & $0 \cdot 34$ & $0 \cdot 21$ & 5 & $\mathrm{R}$ & 56 & $0 \cdot 95$ & $0 \cdot 00$ & 2 & A \\
\hline 24 & $0 \cdot 34$ & 0.70 & 4 & $S$ & 57 & 0.95 & $0 \cdot 00$ & 2 & A \\
\hline 25 & $0 \cdot 34$ & 0.85 & 4 & S & 58 & $0 \cdot 96$ & $0 \cdot 00$ & 2 & A \\
\hline 26 & $0 \cdot 35$ & 0.55 & 4 & $\mathrm{R} \ddagger$ & 59 & $0 \cdot 97$ & $0 \cdot 25$ & 2 & A \\
\hline 27 & 0.39 & $0 \cdot 20$ & 1 & $\mathrm{R}$ & 60 & $0 \cdot 97$ & $0 \cdot 08$ & 2 & A \\
\hline 28 & $0 \cdot 41$ & $0 \cdot 00$ & 1 & $\mathrm{R}$ & 61 & 0.97 & 0.05 & 3 & A \\
\hline 29 & $0 \cdot 43$ & 0.31 & 4 & $\mathrm{R} \ddagger$ & 62 & $0 \cdot 98$ & $0 \cdot 00$ & 1 & A \\
\hline 30 & $0 \cdot 44$ & $0 \cdot 81$ & 2 & $S \ddagger$ & 63 & 0.99 & $0 \cdot 17$ & 2 & A \\
\hline 31 & $0 \cdot 45$ & $0 \cdot 85$ & 4 & $S$ & 64 & $1 \cdot 00$ & $0 \cdot 07$ & 2 & A \\
\hline 32 & $0 \cdot 46$ & 0.37 & 1 & $\mathrm{R}$ & 65 & 1.00 & 0.02 & 1 & A \\
\hline 33 & $0 \cdot 46$ & 0.56 & 3 & $\mathrm{R}$ & 66 & 1.00 & 0.01 & 2 & A \\
\hline
\end{tabular}

${ }^{*}$ Observed response: $1=$ very good; $2=$ good; $3=$ fair $4=$ no change; $5=$ worse.

$\dagger$ Recommended treatment: $P$ (response) $>0 \cdot 60=$ aggressive treatment $(\mathrm{A}) ; P($ no change $)>0 \cdot 60=$ surgery $(\mathrm{S})$; otherwise $=$ radiotherapy $(\mathrm{R})$.

\#'Wrong' decision, based on observed response.

Clearly, the choice of strategy depends on the safety of the proposed treatment. If the treatment has a high risk (e.g. corticosteroids), then only patients with a high probability of response should be treated. But then the untreated patients should be observed for some time, to prevent them from being operated on too soon. On the other hand, if there is minimal risk (e.g. radiotherapy), treating too many patients would be less of a problem. It would then be beneficial to select as many patients as possible with a high probability of inactive disease (prediction of no change) for immediate surgery.

Therefore, we finally used a combination of both models to define a recommended treatment modality for each individual patient, based on both the predicted probabilities of response and no change (Tables 4 and 5). For patients with a predicted probability of response of $>0 \cdot 60$, we recommended aggressive treatment (such as intravenous methylprednisolone pulses), for patients with a predicted probability of no change of $>0 \cdot 60$, we recommended immediate surgery. The remaining patients might then receive a treatment with few side-effects, such as radiotherapy. Based on this strategy and the optimal models, 26 patients were selected for aggressive treatment [of whom 24 (92\%) patients were responders in this study], 20 patients were selected for immediate surgery [of whom 19 (95\%) patients did not change in this study], and 15 patients were selected for radiotherapy [of whom $11(73 \%)$ patients were responders in this study]. In total, we were able to identify the correct treatment (based on a comparison of our recommended treatment with the observed response) in 59/66 (89\%) of the patients. In only one patient (patient 30) would a 'serious' mistake have been made, as this patient would have been recommended for immediate surgery although he/she experienced a good response to radiotherapy in this study.

Unfortunately, several expensive or laborious parameters were included in our optimal models. Using the practical models, we were able to identify the correct treatment in $49 / 66$ (74\%) of patients, 
Table 5. Individual predicted probabilities of response (practical model 1) or no change (practical model 2), observed response, and recommended treatment based on these probabilities

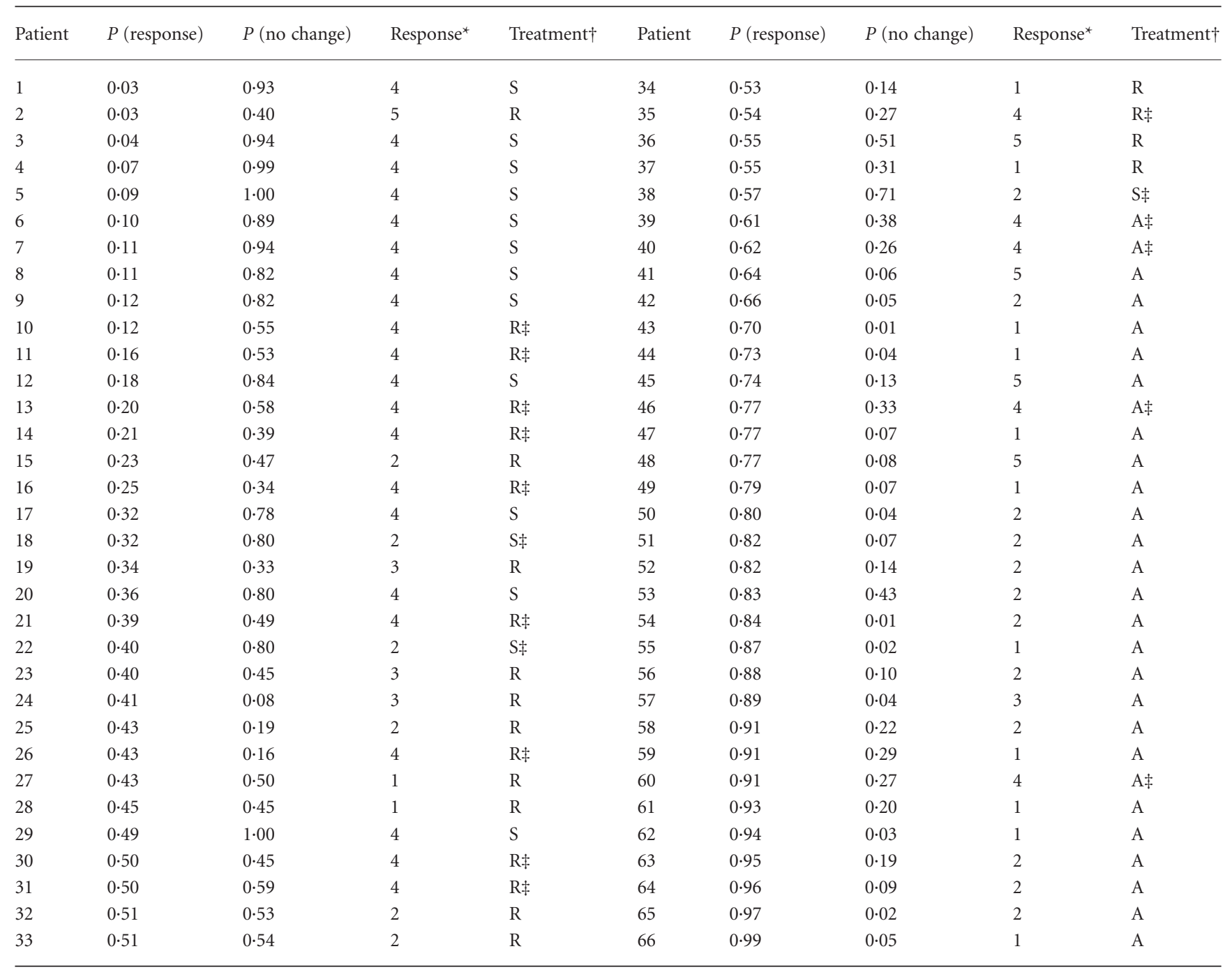

${ }^{*}$ Observed response: 1 = very good; 2 = good; $3=$ fair; $4=$ no change; $5=$ worse.

$\dagger$ Recommended treatment: $P$ (response) $>0 \cdot 60=$ aggressive treatment $(\mathrm{A}) ; P$ (no change) $>0 \cdot 60=$ surgery $(\mathrm{S})$; otherwise $=$ radiotherapy $(\mathrm{R})$.

¥'Wrong' decision, based on observed response.

with three 'serious' mistakes (Table 5). However, in practice these mistakes will not be so 'serious' because there will always be some waiting time due to waiting lists.

Some caution, however, is warranted when these models are used in clinical practice. First, the predicted probabilities of response and no change should be interpreted with caution, as we were not able to calculate confidence intervals around these probabilities. Because of the relative small sample size of our study, the predictive value of some parameters may have been over- or underestimated. In addition, it is well known that predictive models are usually overfit, and that the discriminative ability of these models will probably be less if these models are used in other patient populations. ${ }^{33}$ Therefore, we strongly recommend that our results should be confirmed by other data. Second, all our patients were treated with radiotherapy, thus we only predicted response (or no change) to radiotherapy. Our recommendations for aggressive treatment or surgery were therefore also based on the observed response rates to radiotherapy. Although similar response rates have been observed for other regimens of immunosuppressive treatment, such as corticosteroids, ${ }^{1-5}$ our models should be tested to determine whether they can also predict response to these other treatments. Third, it should be emphasized that these models are based on a population of patients with moderately severe ophthalmopathy. Tests should also be carried out to see whether these models can be applied to patients with mild or severe ophthalmopathy.

In conclusion, we were able to develop two prediction models that seem to be useful in combination to determine a recommendation for treatment for patients with moderately severe ophthalmopathy. Although our findings need to be confirmed by future studies, they are the first evidence for the idea that disease (in)activity should determine which kind of treatment should be used. Further research is warranted to identify simple disease parameters that are predictive of response to immunosuppressive treatment. 


\section{Acknowledgements}

This study was supported by a grant from the Dutch Health Care Insurance Board (OG 94/038).

\section{References}

1 Marcocci, C., Pinchera, A., Prummel, M.F. \& Wiersinga, W.M. (2000) Immunosuppressive management of Graves' ophthalmopathy. In: M.F. Prummel ed. Recent Developments in Graves' Ophthalmopathy. Kluwer, Boston, 101-113.

2 Prummel, M.F. (2001) Immunosuppressive therapy. In: R.S. Bahn ed. Thyroid Eye Disease. Kluwer, Boston, 201-218.

3 Prummel, M.F., Mourits, M.P., Berghout, A., Krenning, E.P., van der Gaag, R., Koornneef, L. \& Wiersinga, W.M. (1989) Prednisone and cyclosporine in the treatment of severe Graves' ophthalmopathy. New England Journal of Medicine, 16, 1403-1405.

4 Prummel, M.F., Mourits, M.P., Blank, L., Berghout, A., Koornneef, L. \& Wiersinga, W.M. (1993) Randomized double-blind trial of prednisone versus radiotherapy in Graves' ophthalmopathy. Lancet, 342 , 949-954.

5 Mourits, M.P., van Kempen-Hartenveld, M.L., Begona Garcia Garcia, M., Koppeschaar, H.P.F., Tick, L. \& Terwee, C.B. (2000) Randomized placebo-controlled study of radiotherapy for Graves' orbitopathy. Lancet, 355, 1505-1509.

6 Rundle, F.F. (1945) Development and course of exophthalmos and ophthalmoplegia in Graves' disease with special reference to the effect of thyroidectomy. Clinical Science, 5, 177-194.

7 Bartalena, L., Pinchera, A. \& Marcocci, C. (2000) Management of Graves' ophthalmopathy: reality and perspectives. Endocrine Reviews, 21, 168-199.

8 Prummel, M.F., Wiersinga, W.M. \& Mourits, M.P. (2000) Assessment of disease activity of Graves' ophthalmopathy. In: M.F. Prummel ed. Recent Developments in Graves' Ophthalmopathy. Kluwer, Boston, 59-80.

9 Bartalena, L., Marcocci, C., Chiovato, L., Laddaga, M., Lepri, G., Andreani, D., Cavallacci, G., Baschieri, L. \& Pinchera, A. (1983) Orbital cobalt irradiation combined with systemic corticosteroids for Graves' ophthalmopathy: comparison with systemic corticosteroids alone. Journal of Clinical Endocrinology and Metabolism, 56, 11391144.

10 Marcocci, C., Bartalena, L., Bogazzi, F., Bruno-Bossio, G., Lepri, A. \& Pinchera, A. (1991) Orbital radiotherapy combined with high dose systemic glucocorticoids for Graves' ophthalmopathy is more effective than radiotherapy alone: results of a prospective randomized study. Journal of Endocrinological Investigation, 14, 853-860.

11 Mourits, M.P., Prummel, M.F., Wiersinga, W.M. \& Koornneef, L. (1997) Clinical Activity Score as a guide in the management of patients with Graves' ophthalmopathy. Clinical Endocrinology, 47, 9-14.

12 Kahaly, G., Forster, G. \& Hansen, C. (1998) Glycosaminoglycans in thyroid eye disease. Thyroid, 8, 429-432.

13 Kahaly, G., Schuler, M., Sewell, A.C., Bernhard, G., Beyer, J. \& Krause, U. (1990) Urinary glycoaminoglycans in Graves' ophthalmopathy. Clinical Endocrinology, 33, 35-44.

14 Hofbauer, L.C., Muhlberg, T., Konig, A., Heufelder, G., Schworm, H.D. \& Heufelder, A.E. (1997) Soluble interleukin-1 receptor antagonist serum levels in smokers and nonsmokers with Graves' ophthalmopathy undergoing orbital radiotherapy. Journal of Clinical Endocrinology and Metabolism, 82, 2244-2247.

15 Wakelkamp, I.M.M.J., Gerding, M.N., van der Meer, J.W.C., Bakker, O.,
Prummel, M.F. \& Wiersinga, W.M. (2000) Both Th1- and Th2derived cytokines in serum are elevated in Graves' ophthalmopathy. Clinical and Experimental Immunology, 121, 453-457.

16 Gerding, M.N., van der Meer, J.W.C., Broenink, M., Bakker, O., Wiersinga, W.M. \& Prummel, M.F. (2000) Association of thyrotrophin receptor antibodies with the clinical features of Graves' ophthalmopathy. Clinical Endocrinology, 52, 267-272.

17 Gerding, M.N., Prummel, M.F. \& Wiersinga, W.M. (2000) Assessment of disease activity in Graves' ophthalmopathy by orbital ultrasonography and clinical parameters. Clinical Endocrinology, 52, 641-646.

18 Prummel, M.F., Suttorp-Schulten, M.S.A., Wiersinga, W.M., Verbeek, A.M., Mourits, M.P. \& Koornneef, L. (1993) A new ultrasonographic method to detect disease activity and predict response to immunosuppressive treatment in Graves' ophthalmopathy. Ophthalmology, 100, 556-561.

19 Hiromatsu, Y., Kojima, K., Ishisaka, N., Tanaka, K., Sato, M., Nonaka, K., Nishimura, H. \& Nishida, H. (1992) Role of magnetic resonance imaging in thyroid-associated ophthalmopathy: its predictive value for therapeutic outcome of immunosuppressive therapy. Thyroid, 2 , 299-305.

20 Prummel, M.F., Gerding, M.N., Zonneveld, F. \& Wiersinga, W.M. (2001) The usefulness of quantitative orbital magnetic resonance imaging in Graves' ophthalmopathy. Clinical Endocrinology, 54, 205-209.

21 Krassas, G.E. \& Kahaly, G. (1999) The role of octreoscan in thyroid eye disease. European Journal of Endocrinology, 140, 373-375.

22 Colao, A., Lastoria, S., Ferone, D., Pivonello, R., Macchia, P.E., Vassallo, P., Bonavolonta, G., Muto, P., Lombardi, G. \& Fenzi, G. (1998) Orbital scintigraphy with [111In-diethylenetriamine pentaacetic acid-D-phe1]-octreotide predicts the clinical response to corticosteroid therapy in patients with Graves' ophthalmopathy. Journal of Clinical Endocrinology and Metabolism, 83, 37903794.

23 Krassas, G.E., Dumas, A., Pontikedes, N. \& Kaltsas, T. (1995) Somatostatin receptor scintigraphy and octreotide treatment in patients with thyroid eye disease. Clinical Endocrinology, 42, 571580.

24 Kahaly, G., Diaz, M., Hahn, K., Beyer, J. \& Bockisch, A. (1995) Indium-111-pentetreotide scintigraphy in Graves' ophthalmopathy. Journal of Nuclear Medicine, 36, 550-554.

25 Mourits, M.P., Prummel, M.F., Wiersinga, W.M. \& Koornneef, L. (1994) Measuring eye movements in Graves' ophthalmopathy. Ophthalmology, 101, 1341-1346.

26 Bahn, R.S. \& Gorman, C.A. (1987) Choice of therapy and criteria for assessing treatment outcome in thyroid-associated ophthalmopathy. Endocrinology and Metabolism Clinics of North America, 16, 391-407.

27 Duke-Elder, S. (1962) The examination of the visual functions. In: S. Duke-Elder ed. The Foundations of Ophthalmopathy: Hereditary, Pathology, Diagnosis, and Therapeutics. System of Ophthalmology, CV Mosby, St Louis, 370-373.

28 Gerding, M.N., Prummel, M.F., Kalmann, R., Koornneef, L. \& Wiersinga, W.M. (1998) The use of colour slides in the assessment of changes in soft-tissue involvement in Graves' ophthalmopathy. Journal of Endocrinological Investigation, 21, 459-462.

29 Mourits, M., Koornneef, L., Wiersinga, W.M., Prummel, M.F., Berghout, A. \& van der Gaag, R. (1989) Clinical criteria for the assessment of disease activity in Graves' ophthalmopathy: a novel approach. British Journal of Ophthalmology, 73, 639-644.

30 Hansen, C., Fraiture, B., Rouhi, R., Otto, E., Forster, G. \& Kahaly, G. 
(1997) HPLC glycosaminoglycan analysis in patients with Graves' disease. Clinical Science (London), 92, 511-517.

31 Gerding, M.N., van der Zant, F.M., van Royen, E.A., Koornneef, L., Krenning, E.P., Wiersinga, W.M. \& Prummel, M.F. (1999) Octreotide-scintigraphy is a disease-activity parameter in Graves' ophthalmopathy. Clinical Endocrinology, 50, 373-379.
32 Hanley, J.A. \& McNeil, B.J. (1982) The meaning and use of the area under a receiver operating characteristics (ROC) curve. Radiology, 143, 29-36.

33 Steyerberg, E.W., Harrell, F.E., Borsboom, G.J.J.M., Eijkemans, M.J.C., Vergouwe, Y. \& Habbema, J.D.F. (2001) Internal validity of predictive models: efficiency of some procedures for logistic regression analysis. Journal of Clinical Epidemiology, 54, 774-781. 